\title{
Extracellular signal-regulated kinase 2 mediates the expression of granulocyte colony-stimulating factor in invasive cancer cells
}

\author{
CHIA-HUEI LEE ${ }^{1}$, SZU-HAN LIN ${ }^{2}$, SHWU-FEN CHANG ${ }^{3}$, PO-YUAN CHANG ${ }^{4}$, \\ ZHI-PING YANG ${ }^{1}$ and SHAO-CHUN LU ${ }^{2}$ \\ ${ }^{1}$ National Institute of Cancer Research, National Health Research Institutes, Zhunan, Miaoli County 35053; \\ ${ }^{2}$ Department of Biochemistry and Molecular Biology, College of Medicine, National Taiwan University, \\ Taipei $10051 ;{ }^{3}$ Graduate Institute of Medical Sciences, Taipei Medical University, Taipei 11051; \\ ${ }^{4}$ Department of Internal Medicine, National Taiwan University Hospital, Taipei 10051, Taiwan, R.O.C.
}

Received February 25, 2013; Accepted April 19, 2013

DOI: $10.3892 /$ or.2013.2463

\begin{abstract}
Granulocyte colony-stimulating factor (G-CSF) affects granulopoiesis and is important for mobilizing neutrophils into blood circulation. Due to the hematopoietic properties of G-CSF, it has been widely used to clinically treat chemotherapy-induced neutropenia. However, G-CSF can promote tumors by inhibiting innate and adaptive immunity and enhancing angiogenesis and neoplastic growth. Most G-CSF-producing tumors are associated with a poor prognosis. This indicates that G-CSF promotes cancer progression. Thus, identifying regulatory molecules involved in tumor-derived G-CSF expression may provide therapeutic targets for cancer treatment. This study identified considerable G-CSF expression in malignant breast, lung and oral cancer cells. However, G-CSF expression was barely detectable in non-invasive cell lines. Expression of G-CSF mRNA and protein increased during exposure to tumor necrosis factor- $\alpha$ (TNF- $\alpha$ ). Treatment with U0126 (a mitogen-activated protein kinase inhibitor) drastically reduced basal levels of G-CSF and TNF- $\alpha$-induced G-CSF in aggressive cancer cells. This study also showed that knockdown of extracellular signal-regulated kinase (ERK) 2 by shRNA was necessary and sufficient to eliminate the expression of tumor-derived G-CSF. This did not apply to ERK1. Therefore, ERK2 (but not ERK1) is responsible
\end{abstract}

Correspondence to: Professor Shao-Chun Lu, Department of Biochemistry and Molecular Biology, College of Medicine, National Taiwan University, No. 1, Sec. 1, Jen-Ai Road, Taipei 10051, Taiwan, R.O.C.

E-mail: 1sc@ntu.edu.tw

Abbreviations: ERK, extracellular signal-regulated kinase; G-CSF, granulocyte colony-stimulating factor; MAPK, mitogen-activated protein kinase; shRNA, short hairpin RNA; TNF- $\alpha$, tumor necrosis factor- $\alpha$

Key words: G-CSF, cancer, MAPK, MEK, ERK2 for the transcriptional regulation of tumor-derived G-CSF. The results indicate the pharmaceutical value of specific ERK2 inhibitors in treating patients with G-CSF-producing tumors.

\section{Introduction}

Granulocyte colony-stimulating factor (G-CSF) stimulates the proliferation, survival and neutrophilic differentiation of hematopoietic progenitor cells, making G-CSF a secreted hematopoietic growth factor that is important for granulopoiesis $(1,2)$. Physiologically, G-CSF affects the mobilization of hematopoietic stem cells, progenitors, and mature cells, particularly neutrophils, into blood circulation (3). Healthy individuals have low levels of G-CSF in serum ranging from $<30$ to $163 \mathrm{pg} / \mathrm{ml}$. By comparison, infection raises G-CSF serum levels to a range of $30-3199 \mathrm{pg} / \mathrm{ml}$ (4). Elevation of G-CSF in serum during infection increases the number of granulocytic progenitor cells and neutrophils, and has highlighted the potential therapeutic value of G-CSF for decades. Administration of recombinant G-CSF has been used clinically to increase the neutrophil count when treating chemotherapyor radiotherapy-induced neutropenia $(5,6)$.

Many clinical reports have documented that leukocytosis occurs in patients with various types of non-hematological malignant tumors with no signs of infection, but higher serum G-CSF produced by the tumor. The G-CSF-producing tumors exhibit significant hyperplastic and metastatic properties and a poor prognosis. Although the pathological significance of G-CSF is poorly known, G-CSF has been reported to induce tumor-cell proliferation and migration $(7,8)$ or induce infiltration of inflammatory cells, which leads to stromal activation providing a neoplastic-promoting microenvironment $(7,9)$. Thus, G-CSF is considered a therapeutic target.

Expression of G-CSF in monocytes and macrophages is induced by IL-1, GM-CSF, TNF- $\alpha$ or LPS (5). However, there is limited knowledge concerning the regulation of G-CSF expression in cancer cells. In this study, we compared the expression levels of G-CSF in various malignant cancer cells and investigated the signaling pathway that is critical for G-CSF expression. The results showed that G-CSF expression 
is highly correlated with the invasiveness of cancer cells, and activation of extracellular signal-regulated kinase (ERK) 2, but not ERK1, is essential for G-CSF expression.

\section{Materials and methods}

Reagents. Dulbecco's modified Eagle's medium (DMEM) and fetal bovine serum (FBS) were obtained from Hyclone Laboratories (Logan, UT, USA). TRIzol reagent and penicillin/streptomycin were obtained from Gibco-BRL Life Technologies (Rockville, MD, USA). MMLV reverse transcriptase was obtained from Promega Corporation (Madison, WI, USA). Pharmacological inhibitors PD98059 and U0126 (MEK inhibitors), LY294002 [a phosphatidylinositol 3-kinase (PI3K) inhibitor], 1L6-hydroxymethyl-chiro-inositol-2-(R)2-O-methyl-3-O-octadecyl-sn-glycerocarbonate (an Akt inhibitor, referred to as Akti in the text), and rapamycin (an mTOR inhibitor) were purchased from Calbiochem (San Diego, CA, USA) and were dissolved in dimethyl sulfoxide (DMSO) which was used at a maximum concentration of $0.1 \%$ in culture medium. Antibodies against Akt, phospho-Akt, $\beta$-actin, ERK1/2, phospho-ERK1/2, p70S6K and phosphop70S6K were purchased from Santa Cruz Biotechnology, Inc. (Santa Cruz, CA, USA).

Cell culture. Human oral squamous cell carcinoma (OSCC) cell lines (HSC-3, SCC-15 and OC3), human breast cancer cell lines (MCF-7, T47D and MDA-MB-231), and human lung adenocarcinoma cell lines (CL1 and CL1-5) (10) were used in this study. HSC-3 cells were grown in DMEM containing 5\% FBS. SCC-15, MCF-7, T47D and MDA-MB-231 cells were grown in DMEM/F12 (1:1 mixture) supplemented with 10\% FBS. OC3 cells were grown in DMEM/KSFM (1:1 mixture) supplemented with 10\% FBS. CL1 and CL1-5 cells were grown in RPMI-1640 supplemented with 5\% FBS. All culture medium contained $2 \mathrm{mM}$ of glutamine and $1,000 \mathrm{U} / \mathrm{ml}$ of penicillinstreptomycin. In these experiments, G-CSF mRNA and protein levels were compared in untreated cells and in cells treated with $10 \mu \mathrm{M}$ of $\mathrm{U} 0126$ for $7 \mathrm{~h}$, unless otherwise specified.

RNA isolation and analysis of G-CSF $m R N A$. Total cellular RNA was isolated from cells using TRIzol reagent. RNA concentrations were estimated from the absorbance at $260 \mathrm{~nm}$. First-strand cDNA was synthesized from total RNA using the MMLV reverse transcriptase and Oligo(dT) primer. The mRNA levels of G-CSF and GAPDH were determined by RT-PCR, using specific primers: 5'-CACTCTGGACAGTGC AGGAAG-3' (F) and 5'-CGACACCTCCAGGAAGCTCTG-3' (R) for G-CSF; 5'-AAAGGATCCACTGGCGTCTTC-3' (F) and 5'-GAATTCGTCATGGATGACCTT-3' (R) for GAPDH. Quantitative real-time PCR (qPCR) was used to quantify the level of G-CSF mRNA. The amplifications were carried out in an ABI Prism 7300 sequence detection system (Applied Biosystems, Foster City, CA, USA). The reaction was performed in a $20-\mu 1$ mixture containing $1 \mathrm{X}$ reaction buffer (SYBRGreen Master Mixture; Applied Biosystems) and $100 \mathrm{nM}$ of the primers: 5'-TCCCCATCCCATGTATTTATCT-3' (F) and 5'-AACTCAGAAATGCAGGGAAGGA-3' (R) for G-CSF; 5'-CATGGCCTTCCGTGTTCCTA-3' (F) and 5'-GC GGCACGTCAGATCCA-3' (R) for GAPDH. The specificity was verified by an additional dissociation curve. The interpolated number of cycles to reach a fixed threshold above background noise $(\mathrm{Ct})$ was used to quantify the amplification.

Western blot analysis. Cellular protein lysates $(20 \mu \mathrm{g}$ of protein/lane) were separated by SDS-PAGE and transferred to a PVDF membrane, which was blocked with 5\% nonfat dried milk in phosphate-buffered saline (PBS) overnight at $4^{\circ} \mathrm{C}$ and then incubated for $1 \mathrm{~h}$ at RT with $0.5 \mu \mathrm{g} / \mathrm{ml}$ of polyclonal antiERK, anti-phospho-ERK or anti- $\beta$-actin $\mathrm{Ab}$ and for $40 \mathrm{~min}$ at $\mathrm{RT}$ with peroxidase-conjugated anti-rabbit IgG $\mathrm{Ab}$. The bound Abs were detected using an improved chemiluminescence detection system (NEN Life Science Products, Boston, MA, USA). Protein concentrations were determined by the Bradford method (DC protein assay; Bio-Rad, Hercules, CA, USA).

Quantitation of G-CSF in culture media. The concentration of human or mouse G-CSF in the culture medium was measured with a human G-CSF Quantikine ELISA kit (R\&D Systems, Minneapolis, MN, USA) according to the manufacturer's instructions.

Transwell invasion assay. An in vitro cell invasion assay was carried out using Transwell chambers $(8-\mu \mathrm{m}$ pore size; BD Biosciences, Franklin Lakes, NJ, USA). The chambers were coated with Matrigel (BD Biosciences). Cells were plated at a density of $5 \times 10^{4}$ cells/chamber in a 24 -well plate containing serum-free DMEM in the upper chamber and DMEM with $10 \%$ FCS in the bottom chamber as the chemoattractant. The cells that had invaded into the bottom chambers were stained with crystal violet at 16 and $24 \mathrm{~h}$ post-seeding for breast and lung cancer cells, respectively. Cells were counted in 4 random fields using MetaMorph software (Molecular Devices, Sunnyvale, CA, USA). Data were averaged for three independent experiments, each with four replicates.

Recombinant lentivirus construction and gene transduction. The pLKO.1-shRNA plasmids encoding an shRNA with a sequence targeting firefly luciferase or with a sequence targeting human ERK1 (5'-CTATACCAAGTCCATCGACAT-3') and ERK2 (5'-CAAAGTTCGAGTAGCTATCAA-3') were obtained from the National RNAi Core Facility at Academia Sinica, Taiwan. These plasmids and lentiviral packaging vectors pMD.G and pCMV- $\Delta$ R8.91 were co-introduced into HEK293T cells by calcium-phosphate transfection method. Viruses were collected from the medium $60 \mathrm{~h}$ after transfection. For knockdown experiments, HSC-3 and MDA-MB-231 cells were infected with the collected viruses over $24 \mathrm{~h}$ in the presence of Polybrene, followed by selection in medium containing puromycin $(10 \mu \mathrm{g} / \mathrm{ml})$ for 7 days.

Statistical analysis. Results are shown as means \pm SD. Differences between mean values were evaluated using the Student's t-test and were considered significant at $\mathrm{p}<0.05$.

\section{Results}

Expression of G-CSF in SCC-15 oral cancer cells is governed by the MEK-ERK signaling pathway. To identify the pathway(s) involved in G-CSF expression in cancer, the 
A

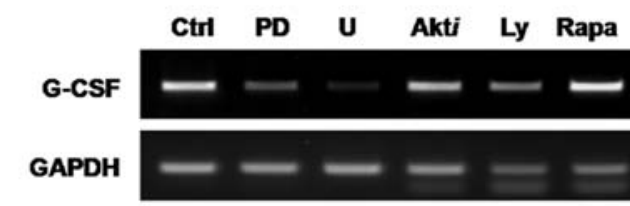

B

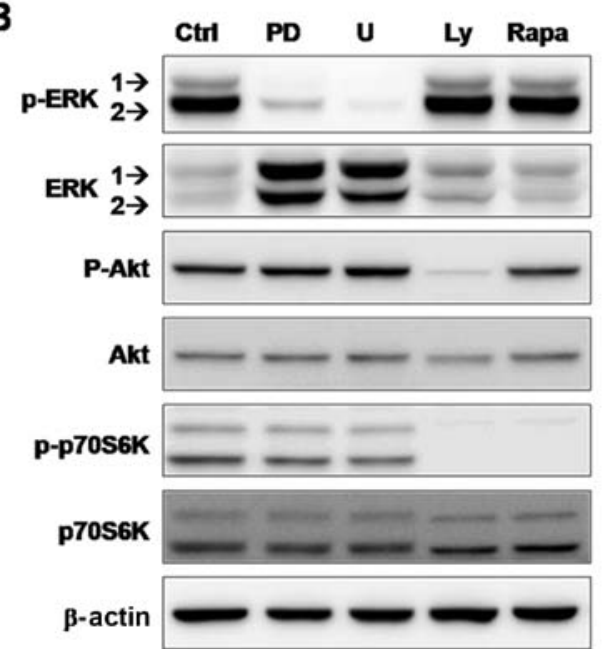

C

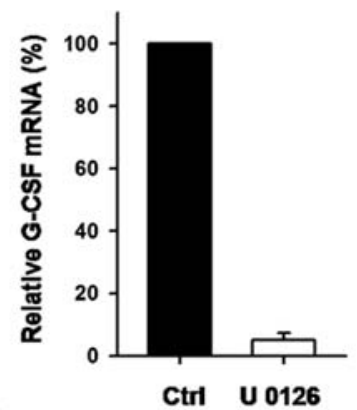

D

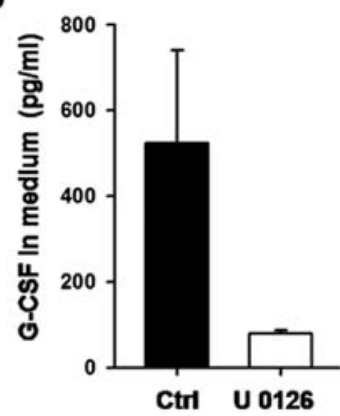

Figure 1. Identification of the signaling pathway regulating the expression of granulocyte colony-stimulating factor (G-CSF) in SCC-15 cells. (A) Cellular total RNAs were harvested from cells treated with PD98059 (PD) and U0126 (U) (40 and $10 \mu \mathrm{M}$, respectively), LY294002 (Ly) (50 $\mu \mathrm{M})$, Akt $i$ (50 $\mu \mathrm{M})$, and rapamycin (Rapa) $(200 \mathrm{ng} / \mathrm{ml})$ for $7 \mathrm{~h}$. Levels of G-CSF and GAPDH mRNA were measured by RT-PCR. (B) After SCC-15 cells were treated with the indicated inhibitor for $7 \mathrm{~h}$, cell lysates were collected and analyzed by western blotting. $\beta$-actin amount was used as a loading control. (C) G-CSF mRNA levels were measured by quantitative RT-PCR with total RNA harvested as described in A. (D) Culture medium was collected from SCC-15 cells treated with U0126 or DMSO for $40 \mathrm{~h}$. The amount of G-CSF protein in the medium was analyzed by ELISA as described. Cells treated with DMSO were used as controls (Ctrl). The values shown are means \pm SD for 3 independent experiments.

effects of various kinase inhibitors on G-CSF expression were examined in SCC-15 cells. As shown in Fig. 1A, a slight decrease in G-CSF mRNA was detected in cells treated with inhibitors of Akt, PI3K or mTOR when compared to the untreated and DMSO-treated control cells. However, G-CSF mRNA was nearly eliminated in cells treated with PD98059 or U0126 (Fig. 1A). The signaling pathways obstructed by the specific inhibitors were verified by western blotting (Fig. 1B). A decrease in phosphorylated ERK1/2 confirmed MEK1/2 inhibition by PD98059 and U0126. Blockage of Akt and p70S6K phosphorylation confirmed PI3K inhibition by Ly294002. Suppression of p70S6K phosphorylation confirmed mTOR inhibition by papamycin. Downregulation of G-CSF mRNA expression in U0126-treated cells was validated by quantitative real-time PCR (Fig. 1C). Moreover, the amount of G-CSF protein decreased significantly after U0126 treatment for $48 \mathrm{~h}$ (Fig. 1D). These results suggest that the expression of G-CSF in SCC-15 cells is regulated through the MEK-ERK signaling pathway.

G-CSF mRNA levels are correlated with ERK1/2 activation and the invasiveness of cancer cells. Since elevated G-CSF levels have been detected in the serum of patients with aggressive tumors, this study examined whether G-CSF expression and ERK1/2 activation are correlated with the invasiveness of cancer cells. Results of the Transwell invasion assay in breast and lung cancer cell lines (Fig. 2A) confirmed previous studies that MDA-MB-231 was the most invasive cell line among the breast cancer cell lines analyzed. In vitro invasiveness was more evident in CL1-5 than in CL1 lung cancer cells. Among these invasive cell lines, G-CSF expression and ERK1/2 activation were detected in the MDA-MB-231 and CL1-5 cells, but not in the less invasive cell lines (Fig. 2B). These results suggest that increased G-CSF expression and ERK1/2 activation may be associated with cancer cell aggressiveness.

Treatment with U0126 drastically inhibits the expression of tumor-derived G-CSF in all cancer cell lines tested. To investigate whether the MAPK pathway is responsible for G-CSF expression in cancer cell lines other than SCC-15, a panel of invasive and G-CSF-producing cancer cells (including MDA-MB-231, CL1-5, OC3 and HSC-3) were treated with U0126. Based on the results of RT-PCR and western blot analyses shown in Fig. 3, the expression of G-CSF mRNA decreased dramatically ( $>90 \%$ inhibition determined by RT-qPCR) when ERK1/2 phosphorylation was inhibited by U0126. These results further emphasize the association between G-CSF expression and ERK1/2 activation in cancer cells, suggesting that ERK1 and/or ERK2 may affect the transcriptional regulation of G-CSF in aggressive cancers.

Blocking MEK activity suppresses TNF- $\alpha$-induced $G$-CSF expression in aggressive breast cancer cells. Chronic inflammation with constitutive production of TNF- $\alpha$ is associated with the steps in tumorigenesis in various types of human cancers (11-13). Koeffler et al (14) reported that G-CSF production is stimulated by TNF- $\alpha$ in fibroblasts, and the authors proposed the involvement of tumor-derived G-CSF in TNF- $\alpha$ - 

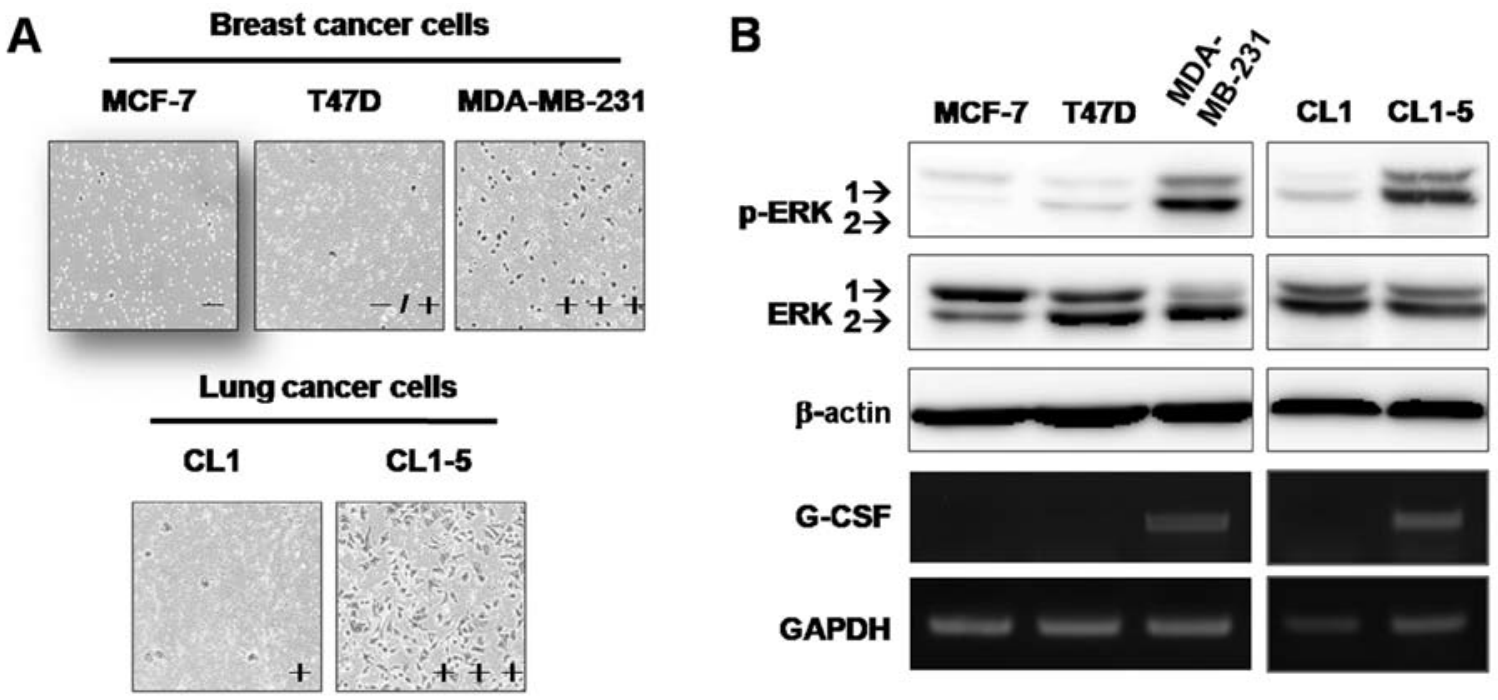

Figure 2. Relationship between expression of granulocyte colony-stimulating factor (G-CSF), MEK activation, and cancer cell invasiveness. (A) The invasiveness of breast and lung cancer cells was evaluated using Matrigel-coated Transwell assay, as described in Materials and methods. Invaded cells were counted in 4 random fields using MetaMorph software. The number of invading cells was the average of 3 independent experiments. Invasiveness of each cell line was expressed as:,$-<10$ invasive cells; -/+, $>10$ and $<50$ invasive cells;,$+>50$ and $<200$ invasive cells; and,$+++>300$ invasive cells. (B) The levels of ERK1/2, phospho-ERK1/2 (p-ERK1/2), and G-CSF mRNA in breast and lung cancer cell lines were measured. Levels of $\beta$-actin protein and GAPDH mRNA were used as internal controls for western blotting and RT-PCR, respectively.

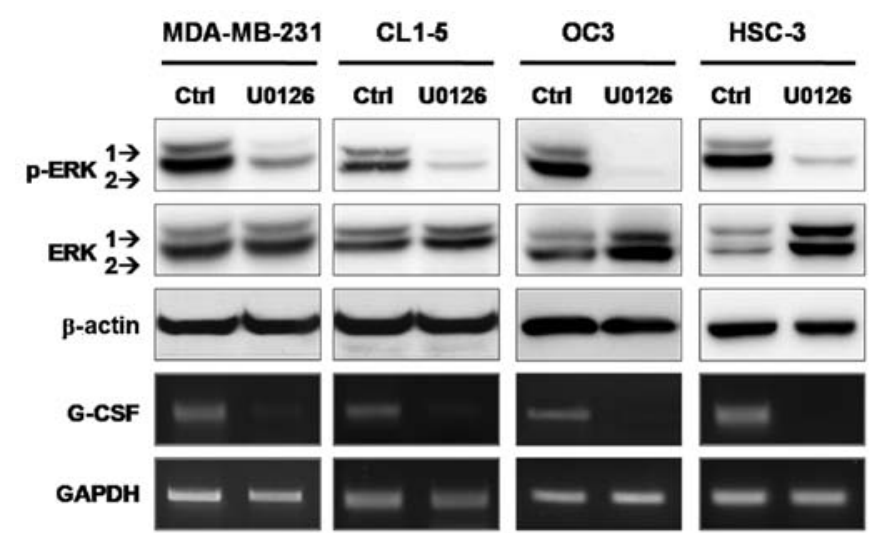

Figure 3. Effects of U0126 treatment on the mRNA levels of granulocyte colony-stimulating factor (G-CSF). Cell lysate and total RNA were harvested from cells treated with DMSO (Ctrl) or U0126 $(10 \mu \mathrm{M})$ for $7 \mathrm{~h}$. Levels of ERK1/2 and p-ERK1/2 were determined by western blotting (upper panels), and G-CSF mRNA levels were determined by RT-PCR (lower panels), with $\beta$-actin and GAPDH used as internal controls.

mediated promotion of oncogenesis. The expression level of G-CSF was relative lower in MDA-MB-231 when compared to the level in the OC 3 and HSC-3 cells. We then tested whether G-CSF production in MDA-MB-231 cells can be further stimulated by TNF- $\alpha$. Results showed a time-dependent increase in G-CSF in culture medium after TNF- $\alpha$ treatment (Fig. 4A), while the TNF- $\alpha$-induced G-CSF production was suppressed when U0126 was added to the cells at either the start of or $12 \mathrm{~h}$ after TNF- $\alpha$ treatment (Fig. 4A). Moreover, the activation of ERK1/2 was analyzed using western blotting to confirm the involvement of the MAPK pathway in TNF- $\alpha$-induced G-CSF expression. The results showed that TNF- $\alpha$ stimulated MEK activation in a time-dependent manner, and phosphorylated ERK1 and ERK2 began to increase as early as $30 \mathrm{~min}$ after

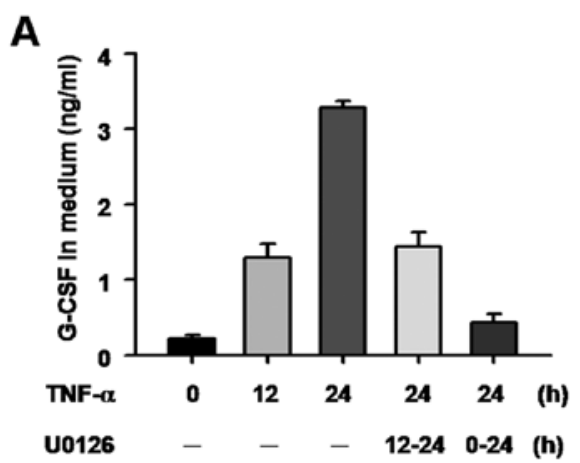

B

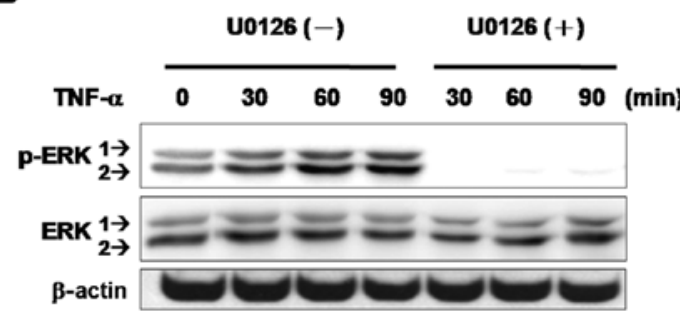

Figure 4. Effects of U0126 and TNF- $\alpha$ treatment on expression of granulocyte colony-stimulating factor (G-CSF) and ERK activation in MDAMB-231 breast cancer cells. (A) MDA-MB-231 cells were grown in a TNF- $\alpha$ $\left(10^{3} \mathrm{U} / \mathrm{ml}\right)$ containing medium with or without $\mathrm{U} 0126(10 \mu \mathrm{M})$ treatment for 12 and $24 \mathrm{~h}$. The amount of G-CSF secreted was determined by ELISA at the times indicated. (B) MDA-MB-231 cells were grown in medium containing TNF- $\alpha\left(10^{3} \mathrm{U} / \mathrm{ml}\right)$ for $90 \mathrm{~min}$ with or without $\mathrm{U} 0126(10 \mu \mathrm{M})$. The amount of ERK1/2 and p-ERK1/2 was determined by western blotting at the times indicated.

TNF- $\alpha$ treatment. These effects were completely inhibited by the presence of U0126 (Fig. 4B). These results suggest that ERK1/2 activation is involved in TNF- $\alpha$-induced G-CSF production in cancer cells. 


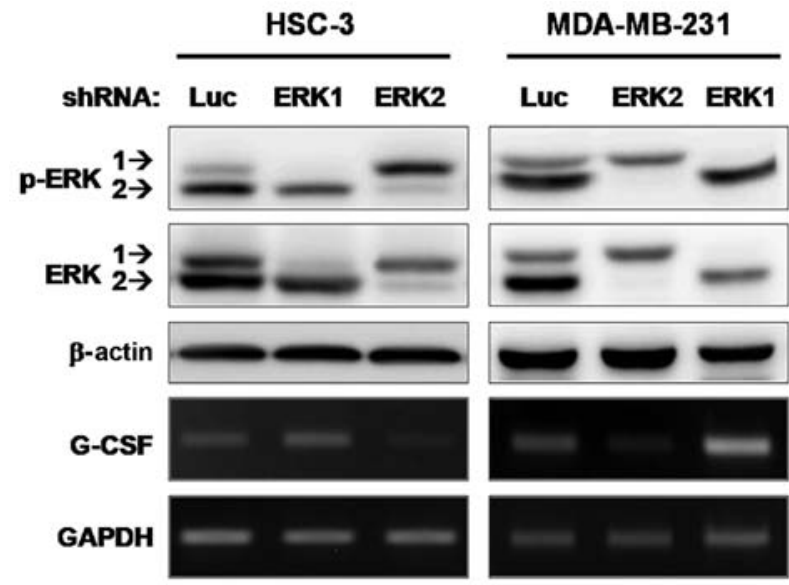

Figure 5. Transcription of tumor-derived G-CSF is regulated by ERK2, but not ERK1. Cell lysate and total RNA were harvested from HSC-3 or MDAMB-231 cells infected with a lentivirus carrying the shRNA of luciferase (Luc), ERK2, or ERK1. Levels of ERK1/2 and p-ERK1/2 were determined by western blotting, and G-CSF mRNA levels were determined by RT-PCR, with $\beta$-actin and GAPDH used as internal controls.

ERK2, and not ERK1, regulates G-CSF expression in cancer cells. MEK1 and MEK2 are dual-specificity kinases that share the consensus kinase motifs of both serine/threonine, and tyrosine kinases. No catalytic substrates for MEK proteins have been identified other than ERK1 and ERK2 (15). To determine whether ERK1 or ERK2 contributes to the transcriptional regulation of G-CSF, an shRNA-knockdown experiment was performed. G-CSF mRNA was determined in HSC-3 and MDA-MB-231 cells (which produce a high level of G-CSF and show invasiveness in vitro) infected with a lentivirus carrying shRNA targeting ERK1 or ERK2. Fig. 5 shows that, in both cell lines, G-CSF expression was almost completely suppressed after shRNA silencing of endogenous ERK2, whereas no significant changes at the mRNA level of G-CSF occurred after ERK1 knockdown. These results demonstrate that ERK2, but not ERK1, is the MAPK implicated in the transcriptional regulation of G-CSF in cancer cells.

\section{Discussion}

Although recombinant G-CSF has been widely used in cancer therapy to ameliorate neutropenia induced by anticancer agents, the deleterious effects of tumor-derived G-CSF have been noted in several studies including clinical case reports as well as in vitro and in vivo experimental studies. The detrimental activities of tumor-produced G-CSF include enhancement of tumor growth, metastasis, and angiogenesis which also creates a tumor-promoting microenvironment. This study found that higher G-CSF expression and ERK activation were associated with the in vitro invasiveness of breast and lung cancer cell lines. The pro-inflammatory cytokine TNF- $\alpha$-induced G-CSF expression in MDA-MB-231 malignant breast cancer cells was suppressed by U0126 treatment accompanied by the blockade of ERK1/2 phosphorylation. Moreover, a similar U0126 effect on G-CSF expression was demonstrated in other invasive cancer cells. An shRNA knockdown approach was used to show that ERK2, but not ERK1, is the main regulator of G-CSF transcription in cancer cells, further demonstrating that the MEK-ERK pathway may affect the regulation of G-CSF expression.

The MAPK pathways are activated by a wide array of extracellular stimuli, which trigger the sequential activation of numerous protein kinases and modulate gene expression, metabolism, cell proliferation, apoptosis and survival. MEK1 and MEK 2 are active in $\sim 30 \%$ of all human cancers where MAPK signaling pathways are implicated (16). Both ERK1 and ERK2 are downstream of MEKs and have been considered interchangeable, since both are similar in sequence, ubiquitously expressed in most tissues, and share upstream activators. However, recent studies have shown that ERK1 and ERK2 are not functionally redundant and exhibit many diverse cellular roles $(17,18)$. The results of this study indicate that inhibition of ERK2 is necessary and sufficient to reduce the level of G-CSF in malignant cancer cells. Carlson et al (19) identified 80 proteins phosphorylated by ERK2. These ERK2 substrates are associated with diverse cellular functions: over 10 proteins are transcription factors or regulators. By modifying these factors, ERK2 may alter the expression of genes such as G-CSF. Thus, the ERK2-related transcription regulators and molecular mechanisms controlling the expression of G-CSF in invasive cancer cells should be further investigated. The association between activated ERK2 and elevated G-CSF levels in cancer cells may partially explain the enhanced neo-angiogenesis and metastasis in tumors with constitutively activated MAPK. Hyper-activated MAPK has been reported in many types of cancers, and several inhibitors are currently under investigation for their potential for targeting the RAS-RAF-MEK-ERK axis and their application as oncology drugs. The findings in this report suggest that targeting ERK2 may be a useful strategy for treating G-CSFproducing cancers.

The expression of G-CSF is controlled at both the transcriptional and post-transcriptional levels. Several transcription factors have been identified, including Oct-1, Oct-2, $\mathrm{C} / \mathrm{EBP} \beta$, and $\mathrm{NF}-\kappa \mathrm{B}$, which bind to the G-CSF promoter and regulate G-CSF transcription. Recently, we reported that inhibiting the PI3K/Akt/mTOR pathway resulted in downregulation of G-CSF expression in LPS- or LTA-induced macrophages $(20,21)$. Rapamycin was found to decrease Oct-2 expression and subsequently downregulate G-CSF expression in macrophages; however, it had no effect on G-CSF expression in SCC-15 cells (Fig. 1). The reason for this is still unclear, although it may be due to the fact that Oct- 2 is expressed mainly in lymphoid and neuronal cells while Oct-1 is sufficient to support G-CSF expression in SCC-15 cells. Transcription factor C/EBP $\beta$, also known as NF-IL6, is involved in controlling cellular proliferation, growth and differentiation. The phosphorylation of $\mathrm{C} / \mathrm{EBP} \beta$ has been shown to be regulated by MEK-ERK signaling (22). We also observed that the interaction of ERK 2 and C/EBP $\beta$ markedly upregulated G-CSF promoter activity (data not shown). NF- $\kappa \mathrm{B}$ is constitutively activated in a range of cancers, and has been demonstrated to promote the development, progression and metastasis of cancers including oral, breast and lung cancers (23-25). Thus, inhibition of NF- $\mathrm{NB}$ and the related signaling pathways may also be another attractive target for cancer therapy. In addition, the level of G-CSF is influenced by its mRNA stability. Multiple AU-rich elements in the $3^{\prime}$ untranslated region of 
G-CSF mRNA imply that mRNA stability can be regulated by p38 MAPK. However, we found that the level of G-CSF mRNA is decreased in some types of cells, but increased in other cell type when treated with SB203580 (a p38-specific inhibitor). Therefore, the role of $\mathrm{p} 38 \mathrm{MAPK}$ in regulating G-CSF mRNA stability requires further investigation.

In summary, this study identified ERK 2 as a major regulator of tumor-derived G-CSF expression and provides evidence to support the use of ERK2 for prognostic purposes in patients with unusually high G-CSF levels. Therapeutic approaches targeting downstream transcription regulators of ERK2 may offer additional strategies for abrogating G-CSF-mediated tumor progression to improve the efficacy of anticancer treatment.

\section{Acknowledgements}

This study was supported by grants from the National Science Council, Taiwan (NSC97-2320-B-002-057-MY3, 99-2320-B-038-009-MY3 and NSC100-2325-B-400-009), National Health Research Institutes, Taiwan (CA-101-PP-01), and Department of Health, Taiwan (DOH101-TD-C-111-004).

\section{References}

1. Welte K, Platzer E, Lu L, Gabrilove JL, Levi E, Mertelsmann R and Moore MA: Purification and biochemical characterization of human pluripotent hematopoietic colony-stimulating factor. Proc Natl Acad Sci USA 82: 1526-1530, 1985.

2. Nicola NA and Metcalf D: Binding of the differentiation-inducer, granulocyte-colony-stimulating factor, to responsive but not unresponsive leukemic cell lines. Proc Natl Acad Sci USA 81: 3765-3769, 1984.

3. Demetri GD and Griffin JD: Granulocyte colony-stimulating factor and its receptor. Blood 78: 2791-2808, 1991.

4. Kawakami M, Tsutsumi H, Kumakawa T, Abe H, Hirai M, Kurosawa S, et al: Levels of serum granulocyte colony-stimulating factor in patients with infections. Blood 76: 1962-1964, 1990.

5. Dale DC: Colony-stimulating factors for the management of neutropenia in cancer patients. Drugs 62 (Suppl 1): 1-15, 2002.

6. Carl W and Havens J: The cancer patient with severe mucositis. Curr Rev Pain 4: 197-202, 2000

7. Obermueller E, Vosseler S, Fusenig NE and Mueller MM: Cooperative autocrine and paracrine functions of granulocyte colony-stimulating factor and granulocyte-macrophage colonystimulating factor in the progression of skin carcinoma cells. Cancer Res 64: 7801-7812, 2004.

8. Mueller MM, Peter W, Mappes M, Huelsen A, Steinbauer H, Boukamp P, et al: Tumor progression of skin carcinoma cells in vivo promoted by clonal selection, mutagenesis, and autocrine growth regulation by granulocyte colony-stimulating factor and granulocyte-macrophage colony-stimulating factor. Am J Pathol 159: 1567-1579, 2001

9. Braun B, Lange M, Oeckler R and Mueller MM: Expression of G-CSF and GM-CSF in human meningiomas correlates with increased tumor proliferation and vascularization. J Neurooncol 68: $131-140,2004$
10. Chu YW, Yang PC, Yang SC, Shyu YC, Hendrix MJ, Wu R, et al: Selection of invasive and metastatic subpopulations from a human lung adenocarcinoma cell line. Am J Respir Cell Mol Biol 17: 353-360, 1997.

11. Egberts JH, Cloosters V, Noack A, Schniewind B, Thon L, Klose S, et al: Anti-tumor necrosis factor therapy inhibits pancreatic tumor growth and metastasis. Cancer Res 68: 1443-1450, 2008.

12. Kulbe H, Thompson R, Wilson JL, Robinson S, Hagemann T, Fatah R, et al: The inflammatory cytokine tumor necrosis factor- $\alpha$ generates an autocrine tumor-promoting network in epithelial ovarian cancer cells. Cancer Res 67: 585-592, 2007.

13. Stathopoulos GT, Kollintza A, Moschos C, Psallidas I, Sherrill TP, Pitsinos EN, et al: Tumor necrosis factor- $\alpha$ promotes malignant pleural effusion. Cancer Res 67: 9825-9834, 2007.

14. Koeffler HP, Gasson J, Ranyard J, Souza L, Shepard M and Munker R: Recombinant human TNF alpha stimulates production of granulocyte colony-stimulating factor. Blood 70: 55-59, 1987.

15. Seger R, Ahn NG, Posada J, Munar ES, Jensen AM, Cooper JA, et al: Purification and characterization of mitogen-activated protein kinase activator(s) from epidermal growth factor-stimulated A431 cells. J Biol Chem 267: 14373-14381, 1992.

16. Hoshino R, Chatani Y, Yamori T, Tsuruo T, Oka H, Yoshida O, et al: Constitutive activation of the 41-/43-kDa mitogen-activated protein kinase signaling pathway in human tumors. Oncogene 18: 813-822, 1999.

17. Vantaggiato C, Formentini I, Bondanza A, Bonini C, Naldini L and Brambilla R: ERK1 and ERK2 mitogen-activated protein kinases affect Ras-dependent cell signaling differentially. J Biol 5: 14, 2006.

18. Yao Y, Li W, Wu J, Germann UA, Su MS, Kuida K and Boucher DM: Extracellular signal-regulated kinase 2 is necessary for mesoderm differentiation. Proc Natl Acad Sci USA 100: 12759-12764, 2003.

19. Carlson SM, Chouinard CR, Labadorf A, Lam CJ, Schmelzle K, Fraenkel E and White FM: Large-scale discovery of ERK2 substrates identifies ERK-mediated transcriptional regulation by ETV3. Sci Signal 4: rs11, 2011.

20. Chou YY and Lu SC: Inhibition by rapamycin of the lipoteichoic acid-induced granulocyte-colony stimulating factor expression in mouse macrophages. Arch Biochem Biophys 508: 110-119, 2011.

21. Chou YY, Gao JI, Chang SF, Chang PY and Lu SC: Rapamycin inhibits lipopolysaccharide induction of granulocyte-colony stimulating factor and inducible nitric oxide synthase expression in macrophages by reducing the levels of octamer-binding factor-2. FEBS J 278: 85-96, 2011.

22. Armstrong DA, Phelps LN and Vincenti MP: CCAAT enhancer binding protein- $\beta$ regulates matrix metalloproteinase-1 expression in interleukin-1 $\beta$-stimulated A549 lung carcinoma cells. Mol Cancer Res 7: 1517-1524, 2009.

23. Coussens LM and Werb Z: Inflammation and cancer. Nature 420: 860-867, 2002.

24. Karin M: Nuclear factor- $\kappa \mathrm{B}$ in cancer development and progression. Nature 441: 431-436, 2006.

25. Huber MA, Azoitei N, Baumann B, Grünert S, Sommer A, Pehamberger $\mathrm{H}$, et al: $\mathrm{NF}-\kappa \mathrm{B}$ is essential for epithelial-mesenchymal transition and metastasis in a model of breast cancer progression. J Clin Invest 114: 569-581, 2004. 